American Journal of Agricultural and Biological Sciences 3 (1): 348-357, 2008

ISSN 1557-4989

(C) 2008 Science Publications

\title{
Biochemical and Genetic Responses of Fungi to the Toxic Effect of Synthetic and Natural Fungicides
}

\author{
${ }^{1,2}$ María-Elena Báez-Flores, ${ }^{1}$ Rosalba Troncoso-Rojas and ${ }^{1}$ Martin-Ernesto Tiznado-Hernández. \\ ${ }^{1}$ Departamento de Tecnología de Alimentos de Orígen Vegetal, Centro de Investigación en \\ Alimentación y Desarrollo, A.C. Carretera a la Victoria Km 0.6, \\ Hermosillo Sonora, México. CP 83000. \\ ${ }^{2}$ Universidad Autónoma de Sinaloa, Facultad de Ciencias Químico Biológicas. Josefa Ortiz de \\ Domínguez esq. Av. de Las Américas, Ciudad Universitaria, Culiacán Sinaloa, México
}

\begin{abstract}
The objective of the present review is to analyze the molecular basis of fungicide resistance mechanism to both synthetic fungicides and the natural fungicides isothiocyanates. The review is focused mainly on Alternaria sp., but whenever available, similar studies in other fungi have been included. Fungal resistant strains to dicarboximide and phenylpyrrole fungicides have been found to contain mutations in one of the proteins involved in the signal transduction pathway that regulates the fungal response to osmotic stress. By the other hand, it was found that isothiocyanates induce enzymes like glutathione S-transferase, cyanide hydratase, heat shock proteins, membrane transporters and proteins associated with the oxidative stress response. Also, inter simple sequence repeats polymorphism was recorded as a response to the isothiocyanates treatment. The knowledge about the genetic basis of the response mechanism of Alternaria sp. to the isothiocyanates is scarce. Therefore, studies by DNA recombinant technology to analyze the fungi responses to fungicides, will allow knowing the metabolic pathways involved in the phenomena to permit the design of strategies to inhibit key reactions involved in the fungal resistance, reaching a better and sustainable fungal infections control.
\end{abstract}

Keywords: iprodione, dicarboximide, fungicide résistance, mutations, gene regulation

\section{INTRODUCTION}

Produce postharvest losses are due to pre-harvest factors ${ }^{[1]}$, poor harvesting techniques ${ }^{[2]}$, bad practices of handling $^{[3]}$ as well as bacterial ${ }^{[4]}$ and fungal infections ${ }^{[5]}$. The amount of fruits and vegetables losses have been estimated to range from 5 to $50 \%$ in developing countries, being still substantial even in areas with the most advanced technologist available ${ }^{[6]}$. In some raw products, fungi are the primary spoilage organisms ${ }^{[5]}$. Out of these, Alternaria spp. is one of the most important postharvest pathogens, as it causes destructive leaf spots, blights (foliar and blossom), blemishes and damage to stored products ${ }^{[7,8]}$. This gender had been reported in apple, broccoli, cauliflower, carrot, potato, cabbage, tomato, pepper, melon, citrics, ornamentals and in a great number of seeds ${ }^{[9]}$.
The main strategy used up to now to control agricultural products infections are mainly based in the use of synthetic chemicals ${ }^{[10]}$. By the years 2000 and 2001, the world use of chemicals in agricultural production was over 5 billions of pounds of which, more than $20 \%$ were used in U.S.A. Out of these, the fungicides represented between 15 and $30 \%{ }^{[11]}$.

A serious consequence of the synthetic chemicals use, is the development of resistant populations ${ }^{[12]}$. This had been reported in fungi ${ }^{[13]}$, insects ${ }^{[14]}$ and weeds ${ }^{[15]}$ and it is an important problem in agriculture, leading to damage on vegetable crops and the chemical's discontinuation or modification of its use ${ }^{[16]}$. The development of a resistant phenotype to a specific chemical is a sophisticated phenomenon that can result from several mechanisms, including: mutations at specific genes ${ }^{[17-21]}$ causing alterations of the drug target, or changes at expression level generating

Corresponding Author: M.E. Tiznado-Hernández, Departamento de Tecnología de Alimentos de Orígen Vegetal, Centro de Investigación en Alimentación y Desarrollo, A.C. Carretera a la Victoria Km 0.6, Hermosillo Sonora, México. CP 83000 
overproduction of the target enzyme, prevention of the entry of the drug at cell membrane level, inhibition of enzymes that convert an inactive drug to its active form and the functioning of a bypass pathway compensating the loss-of-function due to the drug activity, ${ }^{[22]}$. Furthermore, a resistant phenotype can also be due to degradation of toxic compounds to a less toxic chemicals ${ }^{[23-29]}$ and elimination from the cell by efflux pumps ${ }^{[30-32]}$.

In later years, with the objective to carry out a more environmentally friendly fungi control, efforts had been directed towards the study of the effect of natural compounds present in plants, which are thought to be an alternative compatible with the sustainable agriculture concept ${ }^{[10]}$. However, the use of natural compounds is limited and still the use of chemicals is a widespread practice.

Based on above, it is important to elucidate the genetic changes due to the fungicides use in fungal strains that became resistant, in order to develop effective and environmentally friendly strategies to control fungi infections ${ }^{[12]}$. In this review, it was analyzed the recent literature studying the molecular basis of synthetic and natural fungicides resistance in Alternaria gender mainly, although other fungi were included when similar studies were found in the literature.

Resistance phenotype to synthetic fungicides: The development of resistance fungi is an important area of study in population genetics of phytopathogenic fungi because it offers a broad view about how the pathogen population responds to a particular selective pressure. The comparison of the genetic structure between resistant and sensitive subpopulations within the same species, can generate data about the processes of fast evolution involved in the resistance phenomena to the fungicide ${ }^{[33]}$. In general, the development of resistance to a synthetic chemical by a pathogen is a response to the repeated use of a particular fungicide or another one chemically related ${ }^{[34]}$.

Field isolates of different species of Alternaria highly resistant to dicarboximides and phenylpyrroles were recently identified ${ }^{[18]}$. Particularly, the dicarboximide iprodione is widely used as an active ingredient for seed dressing and spray programs to limit outbreaks of diseases on crucifers ${ }^{[19]}$. The appearance of iprodione resistant A. alternata strains was recorded since 1988, less than five years after the release of the fungicide to be used in passion fruit ${ }^{[35]}$. Strains of
Alternaria alternata showing resistance to iprodione had also been reported for other crops. Furthermore, field resistance to this compound had been observed in several fungi ${ }^{[18]}$.

The basis of fungal resistance to iprodione is not well understood, although studies with osmoticsensitive mutants of Neurospora crassa suggest that may be a link between dicarboximide resistance and the regulation of osmotic stress. It had been suggested that this fungicide induces intracellular glycerol accumulation that results in abnormally high internal turgor pressure, leading to the rupture of fungal cells ${ }^{[36]}$. Also, dicarboximides and phenylpyrrole fungicides have been shown to stimulate glycerol synthesis in the wild type $N$. crassa strain, but glycerol synthesis is not induced by these fungicides in the $N$. crassa osmoticsensitive (os mutants $)^{[37]}$, which shows a resistant phenotype to these fungicides. This in turn, provides experimental evidences toward the mode of action of dicarboximides and phenylpyrrole fungicides mentioned above.

The os- 1 gen from $N$. crassa was cloned and found to encode a two component histidine kinase homologous to the Sln1 protein from Sacharomyces cerevisiae $^{[38]}$. A number of $o s-1$ mutants alleles from $N$. crassa have been cloned and shown to contain various mutations in the DNA region encoding one of the six 90 amino acid tandem repeat regions, at the $\mathrm{N}$-terminus of the histidine kinase protein ${ }^{[17]}$. This region is not present in the $\operatorname{Sln} 1$ homolog from S. cerevisiae ${ }^{[39]}$. It had been proposed that these alterations in Os-1 protein function were responsible for the increased resistance to the carboximide and phenylpyrrol fungicides ${ }^{[39]}$. A search of genome databases indicated that most filamentous fungi have the $o s-1$ family histidine kinase genes in their genomes, while dicarboximideinsensitive S. cerevisiae and Schizosaccharomyces pombe do not ${ }^{[40]}$.

In the fungus Cochliobolus heterostrophus, it had been shown that at least three locus were involved in osmosensitivity and resistance to dicarboximide and phenylpyrrole, namely: Dic1, Dic2 and Dic $3^{[20]}$. It was found that the locus Dicl confers both, resistance to dicarboximides and phenylpyrrole fungicides and sensitivity to osmotic stress. Further, it was suggested that fungicide resistance in dicl mutants is caused by alterations in proteins regulating the cellular response to osmotic stress. In another work, a gene encoding a two component histidine kinase protein from $C$. heterostrophus was cloned and sequenced. It was 
demonstrated that this histidine kinase is encoded by Dicl by complementation of the $C$. heterostrophus dicl mutant ${ }^{[21]}$. Moreover, it was found that the predicted protein contained the conserved histidine-kinase domain, the response regulator domain and six repeats of a novel 92-amino acid unit at the N-terminus. Furthermore, the authors found that the osmosensor of C. heterostrophus does not have a membrane-spanning domain, whereas Sln1p of Sacharomyces cerevisisae contains two. These last findings further suggested that the proteins encoded by Dicl (Dic1p) and $\operatorname{Sin} 1(\operatorname{Sln} 1 \mathrm{p})$ regulate response to osmotic stress by different mechanisms of signal transduction.

With the goal to elucidate the molecular basis of the phenotype in each $C$. heterostrophus mutant strain, the mutant dicl alleles were cloned and compared in sequences with the wild-type $\operatorname{strain}^{[21]}$. A fragment isolated from C. heterostrophus by PCR and genomic library screening showed significant homology to the histidine kinase gene encoding $O s-1$ from $N$. crassa; it was denominated BmhkI. In order to determine whether the phenotypes of any of the dicl mutants were conferred by mutations in the Bmhkl gene, mutants strains were transformed with the wild type Bmhkl gene. Representatives of each mutant strain, Dicl (E4504), Dic2 (E4503) and Dic3 (N9005), were used. In the dicl mutant strain E4504, introduction of the wild type Bmhkl reversed the osmosensitivity and fungicide resistant phenotypes, while the phenotypes of Dic2 and Dic3 mutants were not affected. These results suggested that phenotypic alterations observed in dicl mutant strains are due to mutations in the Bmhkl gene. In order to identify the mutations present in dicl gene, genes isolated from 10 mutant strains were sequenced and compared with those from the wild type. Strains E4504, N9006, N9010 and E4509 appear to be null mutant. These strains are highly sensitive to osmotic stress and highly resistant to dicarboximide and phenylpyrrole fungicides suggesting that Diclp is essential for osmotic regulation and resistance to both classes of fungicides.

In the case of the dicl mutant strain E9002, the DNA sequence of Dicl gene revealed that the protein encoded lacked two of the six $\mathrm{N}$-terminal repeats (2 and 4). In dicl mutant strains E9001, N9001 and $\mathrm{N} 9013$ the mutations were located in the N-terminal amino acid repeats of Dic1p resulting in a different single amino acid substitution. The repeats altered were 1,5 and 5 respectively. These mutant strains were highly sensitive to osmotic stress and highly resistant to both dicarboximide and phenylpyrrole fungicides. Therefore, the aminoacid repeat domain of Dic1p may be playing an important function in dicarboximide resistance ${ }^{[21]}$.

It was proposed that the amino acid repeats of the Dic1p protein are the site of action of dicarboximides, while phenylpyrroles may target the kinase region of the protein ${ }^{[41]}$. However, the strains E9003 and N9017 with an amino acid substitution within the kinase domain and the response regulator respectively, were both moderately resistant to both classes of fungicides. Furthermore, strain E9003 was highly sensitive to osmotic stress, while strain N9017 was only slightly affected. The authors suggested that mutations within the kinase and regulator domains affect the signal transduction of the osmosensing pathway in a different way, resulting in variable response to the stress compounds.

The fungal resistance to the carboximide iprodione and the phenylpyrrole fludioxonil, as well as salt tolerance (in order to test their osmotic tolerance) were tested in field strains of Alternaria alternata ${ }^{[18]}$. There were found two isolates Aa 8508 and Aa 8740, that were completely inhibited by $4 \mu \mathrm{g} \mathrm{mL}^{-1}$ of iprodione, while isolates Aa 8501 and Aa 8495 were highly resistant to this fungicide. Similar results were obtained with the phenylpyrrole fungicide fludioxonil. The resistant strains analyzed in this study were isolated from sites which were previously sprayed with the dicarboximide fungicides. Phenotypic testing confirmed that these two isolates showed high level of resistance to iprodione and fludioxonil ${ }^{[18]}$, although with base in their growth characteristics, others authors have suggested that these strains are only moderately resistant $^{[19]}$.

In the test for sensitivity to osmotic stress the mycelial growth behavior of all A. alternata isolates was significantly reduced at $4 \%$ sodium chloride, while the fungicide resistant isolates were only slightly more sensitive to osmotic stress than the sensitive isolates ${ }^{[18]}$. The authors proposed that A. alternata resistance to iprodione was mediated by a mutation in a twocomponent histidine kinase (HK) gene. They amplified a genomic fragment encoding a two component HK protein from an iprodione sensitive field isolates of $A$. alternata showing high homology to previously identified genes from other fungal species, like $N$. crass $^{[42]}$ and Botrytis cinerea ${ }^{[43]}$. A. alternata HK gene, designated $A a H K 1$, was more similar to the HK from $B$. cinerea (Bos1), showing 80\% amino acid identity on 
the sequenced region. The partial amino acid sequence of AaHK1 protein also showed the characteristic protein structure of fungal HK with 6 repeated amino acid domains of approximately 90 amino acids in length followed by a kinase domain ${ }^{[18]}$.

Sequence comparison of fungicide sensitive isolates with fungicide resistant isolates, revealed the presence of a mutation in each one of the resistant isolates. In one fungicide-resistant isolate (Aa 8495), a duplication of an 11 base pairs (bp) sequence was found within the first amino acid repeat domain at bp position 108 , causing a frame shift and resulting in the presence of a premature stop codon at amino acid position 95. In another fungicide resistant isolate (Aa 8501) a $4 \mathrm{bp}$ deletion was detected within the fifth amino acid repeat between bp 1349 and 1352 which also produces a frame shift leading to a termination of the open reading frame at amino acid position 434. In each case, a null mutation was produced. This was the first report of a dicarboximide resistant fungal isolate with a mutation within the first amino acid repeat region ${ }^{[18]}$.

In a recent work, the $A b N I K 1$ gene, encoding a two component histidine kinase protein, was isolated from a wild type dicarboximide and phenylpyrrole sensitive Alternaria brassicicola strain and compared with the corresponding histidine kinase genes from fungicide resistant strains (field and laboratory mutant strains) ${ }^{[19]}$. Mutations were found in the AbNIKl gene in four of five field resistant strains (Abra3, Abra41, Abra7407 and Abra40) and the two laboratory mutants (Abra20M and Abra43M). Furthermore, one of the fungicide resistant field strain (AbraCP), showed no mutation in the AbNlKl gene. Based in this evidences, the fungicide resistant isolates were separated in two groups based on their sensitivity to osmotic stress. The first group showed a moderately osmosensitivity and high fungicide-resistant phenotype (3 field strains (Abra3, Abra41, Abra7407) and 1 laboratory strain (Abra20M); while the second group showed a low osmosensitivity and high fungicide resistant phenotype (one field strains: Abra40 and 1 laboratory strain: Abra43M).

All the strains of the first group had null mutations, resulting from premature translation termination because of a frameshift within repeat 4 , or the presence of stop codons within repeat 5 or near the G-2 box. A mutation within repeat 4 , which consisted of an identical $2 \mathrm{bp}$ deletion leading to a frameshift, was found in two of the strains analyzed: one laboratory resistant strain (Abra20M) and one field resistant strain (Abra41). Among the field resistant strains, in the
Abra40, it was located an amino acid substitution of a glutamic acid at position 753 by a lysine residue within the $\mathrm{H}$ box domain. This strain was highly resistant to fungicides and highly tolerant to osmotic stress. It's likely that this mutation affected significatively the enzyme activity even though it does not lead to any apparent perturbation in the response to osmotic stress. It is possible that a mutation in another locus could confer the fungicide resistant phenotype in Abra40 strain, which is probably the case for the unique strain with no mutation in the AbNIKl gene (AbraCP). Despite of the fact that both strains had a similar phenotype (resistant to fungicide and osmotic stress), the DNA sequence of its $A b N I K 1$ gene did not reveal any difference relative to the wild type.

The laboratory mutant Abra43M was found to have a point mutation that resulted in a stop codon within the repeat 2 . This should be a null mutant, but unlike other A. brassicicola mutants, this strain was not found osmosensitive. The strain Abra43M had a Kozack sequence and a start codon 24 bases downstream from the mutation. The authors assume that this may give rise to a protein lacking the first two amino acid repeat. However, this truncated histidine kinase should still be active and able to regulate the downstream osmosensing pathway.

In addition, histidine kinases from A. brassicicola and $C$. heterostrophus were compared in this work ${ }^{[19]}$. It was found high similarities at the amino acid and nucleotide levels. The first five introns spanning these two coding regions, were found at the same location and only the length of the first intron was different between the two sequences. This difference was mainly due to the presence of a CA $(\mathrm{TA})_{3}$ $\mathrm{CA}(\mathrm{TA})_{3} \mathrm{CA}(\mathrm{TA})_{2} \mathrm{CA}(\mathrm{TA})_{5}$ microsatellite in the sequence of $C$. heterostrophus. Two microsatellites $(\mathrm{GTT})_{7}$ and $(\mathrm{GTT})_{8}$, depending on the $A$. brassicicola strain, were also found in the $3^{\prime}$-untranslated region (3'UTR) of AbNIKI. The authors remarked that the relevance of the presence of these microsatellites in the non-coding region of the two fungal histidine kinase genes is currently unknown. It was hypothesized that the microsatellite expansions in $3^{\prime}$-UTR regions can cause transcription slippage and produce expanded mRNA which can disrupt splicing ${ }^{[44]}$. In agreement with this statement, in human cancer cells, a deletion of $1 \mathrm{bp}$ in a $(\mathrm{T})_{8}$ microsatellite embedded in the $3^{\prime}$-UTR of CEACAMI gene, was identified ${ }^{[45]}$. This gene does not contain any of the regulatory elements already classified. The authors observed that the wild type but not the mutated 3'-UTR, significantly decreased transgene expression at both mRNA and protein level 
suggesting that a single mutation in the $3^{\prime}$-UTR short microsatellite, might affect gene expression.

Steel and Nair ${ }^{[46,47]}$, proposed that dicarboximide resistance in B. cinerea and Alternaria alternata was mediated by altered levels of the antioxidant enzymes catalase (CAT) and superoxide-dismutase (SOD). Experimental evidences supporting this statement were generated by studying the activity levels of CAT and SOD, in a dicarboximide sensible (DS) and dicarboximide resistant (DR) isolate of Botrytis cinere $^{[48]}$. In the absence of the dicarboximide vinclozolin, the DR isolate had 1.5 and 3.0 folds higher CAT and SOD activities, respectively than the DS isolate. In this study, it was observed that the activities of CAT and SOD changed differently in the DS and DR isolates during incubation with vinclozolin. After $1 \mathrm{~h}$ of fungicide treatment, the CAT and SOD activities in the DR isolate increased 46 and $20 \%$, respectively, while in the DS isolate, such increases of the enzyme activities were not obvious. This result implies that trough enhancement the CAT and SOD activities, the dicarboximide resistant isolate could have developed, at least partly, the resistance phenotype. In agreement with these results, Dry and coworkers ${ }^{[18]}$ have speculated that mutations in the two components HK of fungal cells leads to an increase in the endogenous level of anti-oxidant enzymes protecting these cells from the deleterious effects of dicarboximide fungicides. Furthermore, it was hypothesized, based in the work of Choi and coworkers ${ }^{[48]}$ that if the two components HK genes of the fungi above mentioned, carry out a regulatory function, mutations leading to an inactivation of the HK may result in the constitutive induction of stress response proteins such as catalase (CAT) and superoxide dismutase (SOD $)^{[18]}$. In support of this hypothesis, a research carried out by Kovtun and coworkers ${ }^{[49]}$, found the constitutive activation of oxidative stress pathways in high plant cells by a mutation of a specific mitogen activated protein kinase 3 (MAPK3) in Arabidopsis and tobacco cells which activates, in a constitutive manner, the expression of stress response genes such as the anti-oxidant enzyme glutathione-S-transferases (GST).

GSTs are a family of multifunctional enzymes playing an important role in cellular detoxification and excretion of a wide variety of xenobiotic substances, representing an integral part of phase II biotransformation enzymes ${ }^{[50]}$. These enzymes catalyze the sulfur conjugation between the thiol group of GSH (glutamylcysteinylglycine) and an electrophilic moiety in hydrophobic toxicants. Mammalian and plants GSTs have been well characterized, but little is known about fungal GSTs ${ }^{[51]}$. However, it had been suggested that fungal GSTs are more related to mammalian GSTs that to their bacterial counterparts ${ }^{[52]}$. Some of Fungal GSTs studied are GSTs of Aspergillus fumigatus ${ }^{[53]}$, Schizosacharomyces pombe $e^{[54]}, \quad$ S. cerevisiae $^{[55]}$, Issatchenkia orientalis ${ }^{[56]}, \quad$ Phanerochaete chrysosporium $^{[57]}, \quad$ Cunninghamella elegans ${ }^{[52]}$ Streptomyces $_{\text {griseus }}{ }^{[58]}$, Botrytis cinere ${ }^{[59]}$ and Mucor circinelloides $^{[60]}$. Studies undertaken on these fungal GSTs revealed that they are potentially involved in protecting the cell against damage from oxidative stress, xenobiotics, heavy metals and antifungal compounds, thus highlighting the functional diversity of these enzymes ${ }^{[51]}$. Fungal GSTs exhibit differential expression patterns with in such a way that some isoforms had been shown to be induced in the presence of xenobiotics or oxidative stress. In I. orientalis, of two GSTs identified, only one was constitutive and both were induced in the presence of $o$ dinitrobenzene ${ }^{[55,56]}$. In $S$. pombe, three GSTs were induced by oxidative stress, but the transcription of these genes is subjected to differential regulation under various stress conditions ${ }^{[61]}$. Gst1, Gst2 and Gst3 have GST activity with the substrate 1-cloro-2, 4dinitronenzene, while Gst3 has glutathione peroxidase activity. However, all three genes are required for normal cellular resistance to peroxides. Mutants lacking gst 1, gst 2 or $g s t 3$ were more sensitive to the presence of the antifungal drug fluconazole, thereby indicating a role of GST in mediating antifungal drug tolerance ${ }^{[54]}$.

Resistant phenotype to natural fungicides: Among the natural compounds with fungicide activity that are receiving greater attention are the isothiocyanates, mainly due to their potent fungicide activity ${ }^{[62]}$. It has been reported the toxic effect of 3methylsulphinylpropyl-isothiocyanate, bencilisothiocyanate, 2-propenyl-isothiocyanate and (2S)-2hydroxybut-3-enyl-isothiocyanate against eight phytopathogenic fungi: Alternaria alternata, Botrytis cinerea, Rhizoctonia solani, Sclerotinia sclerotiorum, Diaphorthe phaseolorum, Fusarum oxysporum, Colletotricum coccodes and Phitium irregulare ${ }^{[63]}$. Moreover, the effect of isothiocyanates on different developmental stages of isolates of Fusarium oxysporum was evaluated. It was found that both 2propenyl- and ethyl-isothiocyanates inhibited 100 percent the mycelial growth as well as conidial and chlamydospores germination ${ }^{[64]}$.

The activity in vitro and in vivo of bencylisothiocyanate (BITC) on Alternaria alternata strains was evaluated ${ }^{[65]}$. Results of the in vitro experiment showed that a BITC concentration of $0.05 \mathrm{mg} \mathrm{ml}^{-1}$ 
inhibited $90 \%$ of mycelial growth, whereas concentrations of $0.1 \mathrm{mg} \mathrm{ml}^{-1}$ and higher $(0.2-0.4 \mathrm{mg}$ $\mathrm{ml}^{-1}$ ) showed $100 \%$ inhibition. By other side, in the in vivo experiment, the BITC effect at two concentrations (0.28 y $0.56 \mathrm{mg} \mathrm{ml}^{-1}$ ) during 18 and 36 hours on Alternaria alternata infecting tomato fruits was studied. The authors found that a concentration of $0.56 \mathrm{mg} \mathrm{ml}^{-1}$ reduced the fungi infection by Alternaria alternata on tomato fruits by $85 \%$. Further, it was shown that this BITC concentration was more effective than a commercial fungicide in controlling the fungi infection with no negative effects on the postharvest quality of tomato fruit. Another set of experiments found that a concentration of $0.03 \mathrm{mg} \mathrm{ml}^{-1}$ of an isothiocyanate mixture inhibited $100 \%$ the growth of Alternaria alternata under in vitro conditions. Also, the utilization of this isothiocyanate mixture at concentration of 0.56 $\mathrm{mg} \mathrm{ml}^{-1}$ showed a complete inhibition of Alternaria alternata infecting bell pepper fruit. Moreover, it was shown that this treatment with isothiocyanates mixture controlled better the fungi infection than a commercial fungicide without detrimental effects on the postharvest fruit quality ${ }^{[66]}$.

Despite of the fact that the isothiocyanates had shown a clear inhibition activity on fungi growth in experiments carried out under in vivo and in vitro conditions, preliminary experiments carried out in our laboratory, showed that Alternaria alternata is able to grow after being chronically exposed to sublethal dosis of 2-propenyl-isothiocyanate. However, the elimination of the selective pressure along with inoculation in new media render A. alternata showing the same level of sensitivity as the unexposed control. Based on these data, the authors suggested that A. alternata developed an environmental resistance to the toxic effect of the isothiocyanates rather than a resistance developed by mutation, and therefore it dissapeared when the selective pressure was no longer present (R. TroncosoRojas, personal communication).

In order to study the genetic responses of the $A$. alternata to the isothiocyanates, the effect of fungus exposure to 2-propenyl-ITC (2p-ITC) and benzyl-ITC (BITC) on the inter simple sequence repeats regions (ISSR) of A. Alternata genome was analyzed. It was found ISSR polymorphism in the strains exposed to $2 \mathrm{p}$ ITC and BITC when compared with the wild type. In one of the wild type strains, PCR analysis using the oligonucleotide (GACA) ${ }_{4}$ amplified two fragments with sizes of 1.3 and $0.71 \mathrm{~kb}$ whereas in the exposed strain, the $0.71 \mathrm{~kb}$ fragment was not found. It is believed by the authors that the exposition to the isothiocyanates led to the elmination of the small ISSR suggesting an alteration in the microsatellite region GACA. In other strains exposed to 2p-ITC and BITC, it was found two DNA fragments of 603 y $350 \mathrm{bp}$, that were not present in the wild type. Furthermore, in the same experiment, a fragment of $2.17 \mathrm{~kb}$ was found only in the wild type (R. Troncoso-Rojas, personal communication).

The microsatellite regions are one of the most variable elements of the genome ${ }^{[67]}$ and they have been found ubiquitously in the different fungi genome analyzed so $\operatorname{far}^{[68]}$. In average, the mutation rate in the microsatellite regions is various orders of magnitude higher than other DNA regions ${ }^{[69]}$. It had been demonstrated that mutations in the microsatellite regions can be induced by exposition to specific external factors like fungal infection ${ }^{[70]}$, stress by genotoxic chemicals derived from environmental contamination or by climatic change $\mathrm{e}^{[71]}$, and ionizing radiation $^{[72]}$. Therefore, the changes at the microsatellite regions can provide a mechanism for a rapid adaptation to environmental changes in both eukaryotic and prokaryotic organisms ${ }^{[44]}$.

Recently, the effect of 2p-ITC and BITC isothiocyanates on Alternaria brassicicola gene expression was evaluated. Differential display analysis led to the isolation of the first GST gene from this fungus, designed $A b G S t 1$, which was differentially expressed in the presence of 2 -ITC and BITC ${ }^{[51]}$. Furthermore, the authors observed that AbGstl was upregulated during the infection of A. brassicicola on Arabidopsis thaliana, a plant that shows resistance against this pathogen presumably by the presence of isothiocyanates as defense compounds. The deduced amino acid sequence of AbGSt1p showed significant identity to glutathione-S-transferase-I from Saccharomyces cerevisiae and glutathione-transferaseIII from Schizosaccharomyces pombe. The results of this work showing upregulation of AbGstl by isothiocyanates and the increased activity of AbGSt1p in the presence of cyanogenic products strongly suggest that $A b G s t 1$ enables this fungus to tolerate some plant defense compounds ${ }^{[51]}$.

In a more extensive work, Sellam and coworkers ${ }^{[73]}$ studied the changes in gene regulation of $A$. brassicicola to short exposure to $2 \mathrm{p}$-ITC. The authors found upregulation of several genes such as glutathione transferases (GSTs), glutathione peroxidase, $\gamma$ glutamyl-cysteine synthetases, thioredoxins, thioredoxin synthetases, oxidoreductases, (all involved in the oxidative stress response). Moreover, the AllylITC treatment induced one cytochrome P450-encoding gene, 10 genes for membrane transporters and one gene encoding a positive-acting sulphur regulatory protein. 
The authors suggested that $2 p$-ITC treatment generates reactive oxygen species (ROS), leading to the activation of the conserved ITC-detoxification mechanism mediated by phase I and phase II enzymes.

Another gene shown to be involved in isothiocyanate detoxification in Alternaria brassicicola is $C y h A B$, which encodes a cyanide hydratase enzyme. It was shown the upregulation of this gene during the infection of Arabidopsis thaliana by A. brassicicola. Based on these experimental evidences, it was suggested a role for this gene in isothiocyanate detoxification ${ }^{[74]}$.

\section{CONCLUSION}

Data available about the genetic and biochemical mechanisms leading to the development of fungi resistant phenotypes to natural and synthetic fungicides is an imperative need. Also, considering that this phenomenon is the major drawback for the use of fungicides to control fungal infections, it is urgent to conduct more research using techniques able to analyze the genetic responses with tools derived from the DNA recombinant technology. The development of knowledge about the fungal adaptation at genetic level will allow the designing of strategies to control fungi infections more effective and environmentally friendly.

\section{REFERENCES}

1. Snelgar, W., G. HopkirkZ, R. Seelye, P. Martin and P. Manson, 1998. Relationship between Canopy Density and Fruit Quality of Kiwifruit. New Zealand J. of Crop and Horticultural Sci., 26: 223-232

2. Sinha, A., 2002. Harvesting Techniques, Hemi parasites and Fruit Production in Two Non-Timber Forest Tree Species in South India. Forest Ecology and Manage., 168(1): 289-300

3. Clark, C.J., R.B. Jordan, X. Meier and M.A. Manning, 2003. Effect of Mechanical and WaterBased Postharvest Treatments on Storability of Hayward Kiwifruit (Actinidia deliciosa). New Zealand J. of Crop and Horticultural Sci., 31: $247-254$

4. Corbo, M., C. Altieri, D. D'Amato, D. Campaniello, M. Del Nobile and M. Sinigaglia, 2004. Effect of Temperature on Shelf Life and Microbial Population of Lightly Processed Cactus Pear Fruit. Postharvest Biol. Technol., 31(1): 93-104.

5. Barbosa Canovas, G., J.J. Fernandez-Molina, S.M. Alzamora, M.S. Tapia, A. Lopez-Malo and J. Welti
Chanes, 2003. Handling and Preservation of Fruits and Vegetables by Combined Methods for Rural Areas: Technical Manual. pp: 6.

6. Eckert, J. and J. Ogawa, 1985. The Chemical Control of Postharvest Diseases: Subtropical and Tropical Fruits. Annual Review of Phytopathology, 23(1): 421-454.

7. Rotem, J., 1994. The Genus Alternaria: Biology, Epidemiology, and Pathogenicity. APS Press, pp: 1-6.

8. Simmons, E., 1992. Alternaria Taxonomy: Current Status, Viewpoint, Challenge. In: Alternaria Biology, Plant Disease and Metabolites (eds J. C. a. A. Visconti) pp. 1-35. Elsevier Science Publishers, Amsterdam.

9. Thomma, B., 2003. Pathogen Profile Alternaria spp.: from General Saprophyte to Specific Parasite. 4(4): 225- 236.

10. Rosa, E.A. and P.M. Rodrigues, 1999. Towards a More Sustainable Agriculture System: The effect of Glucosinolates on the Control of Soil-Borne Diseases. J. of Horticultural Science and Biotechnology, 74(6): 667-674.

11. Kiely, T., D. Donaldson and A. Grube, 2004. Pesticides Industry Sales and Usage. 2000 and 2001 Market Estimates. USEPA, Office of Prevention, Pesticides, and Toxic Substances www.epa.gov/pesticides

12. Adachi, Y., H. Watanabe and T. Tsuge, 1996. Relationships between Genetic Polymorphisms and Fungicide Resistance within Alternaria alternata. Phytopathology, 86: 1248-1254.

13. Georgopoulos, S., 1987. The development of fungicide resistance. In: Populations of Plant Pathogens: Their Dynamics and Genetics (eds M.S. Wolfe and C.E. Caten) pp: 239-251. Blackwell Scientific Publications, Oxford, UK.

14. Bauer, L., 1995. Resistance: A Threat to the Insecticidal Crystal Proteins of Bacillus thuringiensis. Florida Entomologist, 78(3): 414443.

15. Heap, I., 1997. The Occurrence of HerbicideResistant Weeds Worldwide. Pesticide Science, 51(3): 235-243.

16. Staub, T., 1991. Fungicide Resistance: Practical Experience with Antiresistance Strategies and the Role of Integrated Use. Annual R. of Phytopathology, 29(1): 421-442.

17. Miller, T., S. Renault and C. Selitrennikoff, 2002. Molecular Dissection of Alleles of the Osmotic-1 Locus of Neurospora crassa. Fungal Genetics and Biology, 35(2): 147-55. 
18. Dry, I., K. Yuan and D. Hutton, 2004. Dicarboximide Resistance in Field Isolates of Alternaria alternata Is Mediated by a Mutationin a Two-Component Histidine Kinase Gene. Fungal Genetics and Biology, 41(1): 102-108.

19. Avenot, H., P. Simoneau, B. Iacomi-Vasilescu and N. Bataillé-Simoneau, 2005. Characterization of Mutations in the Two-Component Histidine Kinase Gene AbNIK1 from Alternaria brassicicola That Confer High Dicarboximide and Phenylpyrrole Resistance. Current Genetics, 47(4): 234-243.

20. Yoshimi, A., J. Imanishi, A. Gafur, C. Tanaka and M. Tsuda, 2003. Characterization and Genetic Analysis of Laboratory Mutants of Cochliobolus heterostrophus Resistant to Dicarboximide and Phenylpyrrole Fungicides. J. of General Plant Pathology, 69(2): 101-108

21. Yoshimi, A., M. Tsuda and C. Tanaka, 2004. Cloning and Characterization of the Histidine Kinase Gene Dicl from Cochliobolus heterostrophus That Confers Dicarboximide Resistance and Osmotic Adaptation. Molecular Genetics and Genomics, 271(2): 228-236.

22. Ghannoum, M. A. and L. B. Rice, 1999. Antifungal Agents: Mode of Action, Mechanisms of Resistance, and Correlation of These Mechanisms with Bacterial Resistance. Clinical Microbiology Reviews, 12(4): 501-517.

23. Osbourn, A. E., 1996. Preformed Antimicrobial Compounds and Plant Defense against Fungal Attack. Plant Cell, 8(10): 1821-1831.

24. Morrissey, J. and A.E. Osbourn, 1999. Fungal Resistance to Plant Antibiotics as a Mechanism of Pathogenesis. Microbiology and Molecular Biology Reviews, 63(3): 708-724.

25. Soledade, M., C. Pedras and A.Q. Khan, 1997. Unprecedented Detoxification of the Cruciferous Phytoalexin Camalexin by a Root Phytopathogen. Bioorganic and Medicinal Chemistry Letters, 7(17): 2255-2260.

26. Pedras, M., C. Soledade, A.Q. Khan and J.L. Taylor., 1998. The Phytoalexin Camalexin Is Not Metabolized by Phoma lingam, Alternaria brassiceae, or Phytopathogenic Bacteria. Plant Science, (139): 1-8.

27. Sandrock, R. and H. VanEtten, 1998. Fungal Sensitivity to and Enzymatic Degradation of the Phytoanticipin Alpha-Tomatine. Phytopathology, 88(2): 137-143.

28. Sbaghi, M., P. Jeandet, R. Bessis and P. Leroux, 1996. Degradation of Stilbene-Type Phytoalexins in Relation to the Pathogenicity of Botrytis cinerea to Grapevines. Plant Pathology, 45(1): 139-144.
29. VanEtten, H., R. Sandrock, C. Wasmann, S. Soby, K. McCluskey and P. Wang, 1995. Detoxification of Phytoanticipins and Phytoalexins by Phytopathogenic Fungi. Canadian J. of Botany, 73(S1): 518-525.

30. Alexander, N.J., 1999. TRI12, a Trichotecene Efflux Pump from Fusarium sporotrichoides: Gene Isolation and Expression in Yeast. Molecular and General Genetics 261: 977-984.

31. Callahan, T.M., M.S. Rose, M.J. Meade, M. Ehrenshaft and R.G. Upchurch, 1999. CFP, the Putative Cercosporin Transporter of Cercospora kikuchii, Is Required for Wild Type Cercosporin Production, Resistance, and Virulence on Soybean. Molecular Plant-Microbe Interactions, 12(10): 901910.

32. Del Sorbo, G., H. Schoonbeek and M. De Waard, 2000. Fungal Transporters Involved in Efflux of Natural Toxic Compounds and Fungicides. Fungal Genetics and Biology, 30(1): 1-15.

33. McDonald, B. and J. McDermott, 1993. Population Genetics of Plant Pathogenic Fungi. BioScience, 43(5): 311-319.

34. Brent, K. J., 1995. Fungicide Resistance in Crop Pathogens: How Can it Be Managed? GIFAP Groupement International des Associations Nationales de Fabricants de Produits Agrochimiques, pp: 8.

35. Hutton, D., 1988. The Appearance of Dicarboximide Resistance in Alternaria alternata in Passionfruit in South-East Queensland. Australasian Plant Pathology, 17(2): 34-36.

36. Fujimura, M., N. Ochiai, A. Ichiishi, R. Usami, K. Horikoshi and I. Yamaguchi, 2000. Sensitivity to Phenylpyrrole Fungicides and Abnormal Glycerol Accumulation in $O s$ and Cut Mutant Strains of Neurospora crassa. Nippon Noyaku Gakkaishi, 25(1): 31-36.

37. Zhang, Y., R. Lamm, C. Pillonel, S. Lam and J. $\mathrm{Xu}$, 2002. Osmoregulation and Fungicide Resistance: the Neurospora crassa os-2 Gene Encodes a HOG1 Mitogen-Activated Protein Kinase Homologue. Applied and Environmental Microbiology, 68(2): 532-538.

38. Schumacher, M., 1997. The Osmotic-1 Locus of Neurospora crassa Encodes a Putative Histidine Kinase Similar to Osmosensors of Bacteria and Yeast. Current Microbiology, 34(6): 340-347. 
39. Ochiai, N., M. Fujimura, T. Motoyama, A. Ichiishi, R. Usami, K. Horikoshi and I. Yamaguchi, 2001. Characterization of Mutations in the TwoComponent Histidine Kinase Gene That Confer Fludioxonil Resistance and Osmotic Sensitivity in the Os-1 Mutants of Neurospora crassa. Pest Management Science, 57(5): 437-442.

40. Yamaguchi, I. and M. Fujimura, 2005. Recent Topics on Action Mechanisms of Fungicides. J. of Pesticide Science, 30(2): 67-74.

41. Cui, W., R. Beever, S. Parkes, P. Weeds and M. Templeton, 2002. An Osmosensing Histidine Kinase Mediates Dicarboximide Fungicide Resistance in Botryotinia fuckeliana (Botrytis cinerea). Fungal Genetics Biology, 36(3): 187-198.

42. Alex, L., K. Borkovich and M. Simon, 1996. Hyphal Development in Neurospora crassa: Involvement of a Two-Component Histidine Kinase. Proceedings of the National Acad Sci. of the United States of America 93: 3416-3421.

43. Oshima, M., M. Fujimura, S. Banno, C. Hashimoto, T. Motoyama, A. Ichiishi and I. Yamaguchi, 2002. A Point Mutation in the TwoComponent Histidine Kinase $\mathrm{BcOS}-1$ Gene Confers Dicarboximide Resistance in Field Isolates of Botrytis cinerea. Phytopathology, 92(1): 75-80.

44. Li, Y., A. Korol, T. Fahima and E. Nevo, 2004. Microsatellites Within Genes: Structure, Function, and Evolution. Molecular Biology and Evolution, 21(6): 991-1007.

45. Ruggiero, T., 2003. Deletion in a $(\mathrm{T})_{8}$ Microsatellite Abrogates Expression Regulation by 3'-UTR. Nucleic Acids Research, 31(22): 65616569.

46. Steel, C. and N. Nair, 1993. The Physiological Basis of Resistance to the Dicarboximide Fungicide Iprodione in Botrytis cinerea. 47(1): 6068.

47. Steel, C. and N. Nair, 1995. Oxidative Protective Mechanisms and Resistance to the Dicarboximide Fungicide, Iprodione, in Alternaria alternata. 143(9): 531-535.

48. Choi, G. J., H. J. Lee and K. Y. Cho, 1997. Involvement of Catalase and Superoxide Dismutase in Resistance of Botrytis cinerea to Dicarboximide Fungicide Vinclozolin. Pesticide Biochemistry and Physiology, 59: 1-10.

49. Kovtun, Y., W. Chiu, G. Tena and J. Sheen, 2000. Functional Analysis of Oxidative Stress-Activated Mitogen-Activated Protein Kinase Cascade in Plants. Proceedings of the National Academy of Sciences of the United States of America, 97( 6 ): 2940-2945.
50. Eaton, D. L. and T. K. Bammler, 1999. Concise Review of the Glutathione S-transferases and Their Significance to Toxicology. Toxicological Sciences, (49): 156-164.

51. Sellam, A., P. Poupard and P. Simoneau, 2006. Molecular Cloning of AbGstl Encoding a Glutathione Transferase Differentially Expressed During Exposure of Alternaria brassicicola to Isothiocyanates. FEMS Microbiology Letters, 258: 241- 249.

52. Cha, C.J., S.J. Kim, Y.H. Kim, R. Stingley and C.E. Cerniglia, 2002. Molecular Cloning, Expression and Characterization of a Novel Glutathione S-Transferase Class from the Fungus Cunninghamella elegans. Biochemical J., 368: 589-595.

53. Burns, C., R. Geraghty, C. Neville, A. Murphy, K. Kavanagh and S. Doyle, 2005. Identification, Cloning, and Functional Expression of Three Glutathione Transferase Genes from Aspergillus fumigatus. Fungal Genetics and Biology, 42(4): 319-327.

54. Veal, E. A., W. M. Toone, N. Jones and B. A. Morgan, 2002. Distinct Roles for Glutathione Stransferases in the Oxidative Stress Response in Schizosaccharomyces pombe. J. of Biological Chemistry, 277: 35523-35531.

55. Choi, J. H., W. Lou and A. Vancura, 1998. A Novel Membrane-bound Glutathione S-transferase Functions in the Stationary Phase of the Yeast Saccharomyces cerevisiae. The J. of Biological Chemistry 273( 45 ): 29915-29922.

56. Tamaki, H., K. Yamamoto and H. Kumagai, 1999. Expression of Two Glutathione S-Transferase Genes in the Yeast Issatchenkia orientalis Is Induced by o-Dinitrobenzene during Cell Growth Arrest. J. of Bacteriology, 181(9): 2958-2962.

57. Dowd, C. A., C. M. Buckley and D. Sheehan, 1997. Glutathione S-transferases From the WhiteRot Fungus, Phanerochaete chrysosporium. Biochemical J., 324(1): 243-248.

58. Dhar, K., A. Dhar and J. Rosazza, 2003. Glutathione S-Transferase Isoenzymes from Streptomyces griseus. Applied and Environmental Microbiology, 69(1): 707-710.

59. Prins, T., L. Wagemakers, A. Schouten and J. van Kan, 2000. Cloning and Characterization of a Glutathione S-transferase Homologue from the Plant Pathogenic Fungus Botrytis cinerea. Molecular Plant Pathology, 1(3): 169-178. 
60. Dowd, C.A. and D. Sheehan, 1999. Variable Expression of Glutathione S-transferase Isoenzymes in the Fungus Mucor circinelloides. FEMS Microbiology Letters, 170: 13-17.

61. Kim, H., B. Kim, E. Park, K. Ahn and C. Lim, 2004. Differential Regulation of Three Genes Encoding Glutathione S-Transferases in Schizosaccharomyces pombe. Molecules and cells, 18(3): 332-339.

62. Mayton, H., C. Olivier, S. Vaughn and R. Loria, 1996. Correlation of Fungicidal Activity of Brassica Species with Allyl Isothiocyanate Production in Macerated Leaf Tissue. Phytopathology, 86(3): 267-271.

63. Manici, L., L. Lazzeri and S. Palmieri, 1997. In Vitro Fungitoxic Activity of Some Glucosinolates and Their Enzyme-Derived Products toward Plant Pathogenic Fungi. J. of Agricultural and Food Chemistry, 45(7): 2768-2773.

64. Smolinska, U., M. Morra, G. Knudsen and R. James, 2003. Isothiocyanates Produced by Brassicaceae Species as Inhibitors of Fusarium oxysporum. Plant Disease, 87(4): 407-412.

65. Troncoso-Rojas, R., A. Sánchez-Estrada, C. Ruelas, H.S. García and M. Tiznado-Hernández, 2005. Effect of Benzyl Isothiocyanate on Tomato Fruit Infection Development by Alternaria alternata. J. of the Science of Food and Agriculture, 85(9): 1427-1434.

66. Ruelas, C., M.E. Tiznado-Hernández, A. SánchezEstrada, M.R. Robles-Burgueño and R. TroncosoRojas, 2006. Changes in Phenolic Acid Content During Alternaria alternata Infection in Tomato Fruit. J. of Phytopathology, 154(4 ): 236-244.
67. Weber, J., 1990. Informativeness of Human (dCdA) n.(dG-dT) n Polymorphisms. Genomics, 7(4): 524-30.

68. Lim, S., L. Notley-McRobb, M. Lim and D. Carter, 2004. A Comparison of the Nature and Abundance of Microsatellites in 14 Fungal Genomes. Fungal Genetics and Biology, 41(11): 1025-1036.

69. Nikitina, T. and S. Nazarenko, 2004. Human Microsatellites: Mutation and Evolution. Russian J. of Genetics, 40(10): 1065-1079.

70. Schmidt, A. and V. Mitter, 2004. Microsatellite Mutation Directed by an External Stimulus. Mutation Research, 568(2): 233-43.

71. Hoffman, P., J. Leonard, G. Lindberg, S. Bollmann and J. Hays, 2004. Rapid Accumulation of Mutations During Seed-to-Seed Propagation of Mismatch-Repair-Defective Arabidopsis. Genes and Development, 18(21): 2676-2685.

72. Vornam, B., O. Kuchma, N. Kuchma, A. Arkhipov and R. Finkeldey, 2004. SSR Markers as Tools to Reveal Mutation Events in Scots Pine (Pinus sylvestris L.) from Chernobyl. European J. of Forest Research, 123(3): 245-248.

73. Sellam, A., A. Dongo, T. Guillemette, P. Hudhomme and P. Simoneau, 2007. Transcriptional Responses to Exposure to the Brassicaceous Defence Metabolites Camalexin and Allyl-Isothiocyanate in the Necrotrophic Fungus Alternaria brassicicola. Molecular Plant Pathology, 8(2): 195-208.

74. Cramer, R.A. and C.B. Lawrence, 2004. Identification of Alternaria brassicicola Genes Expressed in Planta During Pathogenesis of Arabidopsis thaliana. Fungal Genetics and Biology, 41(2): 115-128. 